\title{
ON THE STRUCTURE OF SMOOTH COMPONENTS OF SPRINGER FIBERS
}

\author{
LUCAS FRESSE, ANNA MELNIKOV, AND SAMMAR SAKAS-OBEID \\ (Communicated by Lev Borisov)
}

\begin{abstract}
The aim of this paper is to study the structure of the smooth irreducible components of the Springer fibers associated to nilpotent endomorphisms of nilpotency order 2. Relying on its combinatorial interpretation in terms of standard Young tableaux, we show that each smooth component has a structure of iterated bundle of Grassmannian varieties, with explicit base. Using this description, we then classify the smooth components according to their Poincaré polynomials.
\end{abstract}

\section{INTRODUCTION}

Let $V$ be a complex vector space of finite dimension $n \geq 1$. There are several elementary algebraic projective varieties that one can attach to $V$. We mention three of them.

- The projective space $\mathbb{P}(V)$ is the set of lines in $V$. We abbreviate $\mathbb{P}^{n}:=$ $\mathbb{P}\left(\mathbb{C}^{n+1}\right)$ so that $\operatorname{dim} \mathbb{P}^{n}=n$.

- More generally, the Grassmannian variety $G r_{d}(V)$ is the set of $d$-dimensional subspaces of $V$. We abbreviate $G r_{d}(n):=G r_{d}\left(\mathbb{C}^{n}\right)$.

- The flag variety $\mathcal{F}(V)$ is the set of complete flags, i.e., sequences of subspaces $\left(V_{0} \subset V_{1} \subset \ldots \subset V_{n}=V\right)$ such that $\operatorname{dim} V_{i}=i$ for all $i$. We abbreviate $\mathcal{F}(n):=\mathcal{F}\left(\mathbb{C}^{n}\right)$.

These varieties are elementary in the sense that they are smooth, irreducible, endowed with a natural transitive action of the linear group $G L(V)$, and they admit natural cell decompositions from which their cohomology spaces can easily be computed (see Section 2.3).

In this paper, we consider a fourth example of variety. Given a nilpotent endomorphism $u: V \rightarrow V$, we let

$$
\mathcal{F}_{u}=\left\{\left(V_{0}, V_{1}, \ldots, V_{n}\right) \in \mathcal{F}(V): u\left(V_{i}\right) \subset V_{i} \quad \forall i=1, \ldots, n\right\},
$$

which is a projective subvariety of $\mathcal{F}(V)$ called a Springer fiber as it coincides with the fiber over $u$ of the Springer resolution (see [12]). The variety $\mathcal{F}_{u}$ is not an elementary one: it is reducible in general and its irreducible components may have a complicated structure; in particular, they may be singular and it is in general difficult to determine their cohomology.

Received by the editors September 20, 2013 and, in revised form, December 15, 2013.

2010 Mathematics Subject Classification. Primary 14M15, 17B08, 05E10.

Key words and phrases. Components of a Springer fiber, Grassmannian varieties, flag manifolds, iterated bundles, Betti numbers.

The first author was partially supported by ISF grant 882/10. 
In fact, the structure of the variety $\mathcal{F}_{u}$ strongly depends on (and only on) the Jordan form of $u$. In the particular cases where $u$ has only one nontrivial Jordan block or $u$ has only two blocks, it is known from Fung [7] and Vargas [13] that all the irreducible components of $\mathcal{F}_{u}$ are smooth, and in fact it was shown in [7] that in these cases every component is an iterated bundle of Grassmannian varieties.

Our general purpose is to understand the structure of the components of $\mathcal{F}_{u}$, at least of the smooth components of $\mathcal{F}_{u}$, in more cases.

In this paper, we focus on the case where $u^{2}=0$. This is a case where $\mathcal{F}_{u}$ admits singular components and, up to now, this is the only such case where we dispose of a combinatorial criterion specifying whether a component is smooth or singular; this criterion is recalled in Theorem 2 In this paper, we prove the following:

Theorem 1. Assume that $u^{2}=0$. Then, every smooth irreducible component of $\mathcal{F}_{u}$ is an iterated bundle of Grassmannian varieties.

In fact, once we introduce the combinatorial parametrization of components (see Subsection 2.1), we are able to state a more precise statement (Theorem 3) which indicates the explicit base of the iterated bundle and from which we deduce formulas for Betti numbers and Poincaré polynomials. As a complement, we obtain a classification of the smooth components according to their Poincaré polynomials (Theorem 4).

Our study relies on the specificity of the case where $u^{2}=0$, which was pointed out in 6]. For $u$ more general, it is hard to understand the structure of all the smooth components of $\mathcal{F}_{u}$. We however mention the works [1], [5], 8] where the authors construct certain special families of smooth components occurring in each Springer fiber $\mathcal{F}_{u}$ and which are also iterated bundles of Grassmannian varieties.

In view of our result and the above comments, it is natural to ask whether one can find smooth components of Springer fibers that cannot be described as iterated bundles of Grassmannian varieties.

\section{Statement of the Results}

2.1. Springer fibers and their irreducible components. In this section, we present general properties of the Springer fiber $\mathcal{F}_{u}$ defined in Section 1, for $u: V \rightarrow$ $V$ a nilpotent endomorphism.

First note that, for $g \in G L(V)$, the automorphism $g: \mathcal{F}(V) \rightarrow \mathcal{F}(V)$ given by $g\left(V_{0}, V_{1}, \ldots, V_{n}\right)=\left(g\left(V_{0}\right), g\left(V_{1}\right), \ldots, g\left(V_{n}\right)\right)$ restricts to an isomorphism of algebraic varieties $g: \mathcal{F}_{u} \rightarrow \mathcal{F}_{g u g^{-1}}$. This implies that the variety $\mathcal{F}_{u}$ is essentially characterized by the Jordan form $J(u)$ of $u$. Since 0 is the only eigenvalue of $u$, this Jordan form can be identified with the sequence $J(u):=\lambda=\left(\lambda_{1} \geq \ldots \geq \lambda_{k}\right)$ of the sizes of the Jordan blocks of $u$ written in nonincreasing order. This sequence is a partition of $n$ (which we denote $\lambda \vdash n$ ). We write $\mathcal{F}_{\lambda}:=\mathcal{F}_{u}$ for such $u$.

In two opposite extremal cases, this variety is irreducible: if $u$ is regular, in other words $\lambda=(n)$, then $\mathcal{F}_{(n)}:=\mathcal{F}_{u}$ consists of the single complete flag $\left(V_{0}, V_{1}, \ldots, V_{n}\right)$ where $V_{i}=\operatorname{ker} u^{i}$. If $u=0$, so $\lambda=\left(1^{n}\right)$, then $\mathcal{F}_{\left(1^{n}\right)}:=\mathcal{F}_{0}$ coincides with the whole flag variety $\mathcal{F}(n)$. In any other case, $\mathcal{F}_{\lambda}$ is reducible.

For a general partition $\lambda=\left(\lambda_{1} \geq \ldots \geq \lambda_{k}\right) \vdash n$, the dimension of $\mathcal{F}_{\lambda}$ and the decomposition of $\mathcal{F}_{\lambda}$ into irreducible components can be combinatorially expressed in terms of Young diagrams and standard Young tableaux accordingly. This expression is recalled in what follows. First, the partition $\lambda$ can be represented by 
the Young diagram $Y_{\lambda}$, which is an array of $k$ left-justified rows of empty boxes with the $i$-th row containing $\lambda_{i}$ boxes. Let $\lambda^{*}=\left(\lambda_{1}^{*}, \ldots, \lambda_{\ell}^{*}\right)$ denote the conjugate partition, that is, the list of the lengths of the columns in $Y_{\lambda}$. By [11, §II.5.5], the dimension of $\mathcal{F}_{\lambda}$ is given by the formula

$$
\operatorname{dim} \mathcal{F}_{\lambda}=\sum_{i=1}^{\ell} \frac{\lambda_{i}^{*}\left(\lambda_{i}^{*}-1\right)}{2}
$$

Example 1. If $\lambda=(2,2,1)$, then $Y_{\lambda}=\square$ and $\lambda^{*}=(3,2)$, so $\operatorname{dim} \mathcal{F}_{\lambda}=4$.

A standard tableau $T$ of shape $\lambda$ is by definition a numbering of the boxes of $Y_{\lambda}$ by the numbers $1, \ldots, n$ such that the entries are increasing along the rows (from left to right) and the columns (from top to bottom). We denote by $\mathbf{T a b}_{\lambda}$ the set of all standard tableaux of shape $Y_{\lambda}$. For $i \in\{1, \ldots, n\}$, let $T^{i} \subset T$ denote the subtableau formed by the entries $1, \ldots, i$ and let $\lambda^{T^{i}} \vdash i$ be its shape, i.e., the partition such that $T^{i} \in \mathbf{T a b}_{\lambda^{T^{i}}}$. Then, the standard tableau $T$ is equivalent to the datum of the increasing sequence of partitions $\left(\lambda^{T^{1}}, \lambda^{T^{2}}, \ldots, \lambda^{T^{n}}=\lambda\right)$; this sequence is increasing in the sense that the corresponding Young diagrams $Y_{\lambda^{T^{1}}} \subset Y_{\lambda^{2}} \subset \ldots \subset Y_{\lambda^{T^{n}}}=Y_{\lambda}$ are in increasing order for the inclusion (i.e., the length of the $j$-th row of $Y_{\lambda^{T^{i}}}$ is less than or equal to the length of the $j$-th row of $Y_{\lambda^{T^{i+1}}}$ for all $i \in\{1, \ldots, n-1\}$, all $j \geq 1$ ).

Note that an element $F=\left(V_{0}, V_{1}, \ldots, V_{n}\right) \in \mathcal{F}_{u}=\mathcal{F}_{\lambda}$ is by definition a sequence of $u$-stable subspaces, hence for all $i \in\{1, \ldots, n\}$ we can consider the restriction $\left.u\right|_{V_{i}}: V_{i} \rightarrow V_{i}$ which is a nilpotent endomorphism. Its Jordan form $J\left(\left.u\right|_{V_{i}}\right)$ is a partition of $i$ and the sequence of partitions $\left(J\left(\left.u\right|_{V_{i}}\right)\right)_{i=1}^{n}$ is increasing in the above sense. Setting

$$
\mathcal{F}_{u}^{T}=\left\{\left(V_{0}, \ldots, V_{n}\right) \in \mathcal{F}_{\lambda}: J\left(\left.u\right|_{V_{i}}\right)=\lambda^{T^{i}} \forall i=1, \ldots, n\right\},
$$

we get a decomposition $\mathcal{F}_{u}=\bigsqcup_{T \in \mathbf{T a b}_{\lambda}} \mathcal{F}_{u}^{T}$ parameterized by the standard tableaux of shape $\lambda$. By [11, §II.5.4-5], for each $T$, the set $\mathcal{F}_{u}^{T}$ is a locally closed, irreducible subset of $\mathcal{F}_{u}$, and $\operatorname{dim} \mathcal{F}_{u}^{T}=\operatorname{dim} \mathcal{F}_{u}$. Define $\mathcal{F}_{T}:=\overline{\mathcal{F}_{u}^{T}}$ to be the closure in Zariski topology. Then:

Proposition 1. $\left\{\mathcal{F}_{T}\right\}_{T \in \mathbf{T a b}_{\lambda}}$ is the set of all irreducible components of $\mathcal{F}_{\lambda}=\mathcal{F}_{u}$. Moreover, we have $\operatorname{dim} \mathcal{F}_{T}=\operatorname{dim} \mathcal{F}_{\lambda}$ for all $T$.

Example 2. In Example 1, $\mathcal{F}_{\lambda}$ has five irreducible components corresponding to

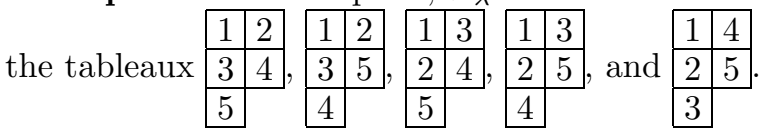

2.2. On irreducible components of $\mathcal{F}_{\lambda}$ for $\lambda=(n-k, k)^{*}$. In this paper, we focus on Springer fibers $\mathcal{F}_{\lambda}=\mathcal{F}_{u}$ attached to nilpotent endomorphisms such that $u^{2}=0$ or, equivalently, attached to partitions of the form $\lambda=(n-k, k)^{*}$ with $1 \leq k \leq n-k$. Equivalently, the Young diagram $Y_{\lambda}$ has two columns of lengths $n-k, k$ and thus (see (10)

$$
\operatorname{dim} \mathcal{F}_{\lambda}=\frac{(n-k)(n-k-1)}{2}+\frac{k(k-1)}{2} .
$$


In this situation, the irreducible components $\mathcal{F}_{T} \subset \mathcal{F}_{\lambda}$ may be singular, and the characterization of the smooth and the singular components is known: we refer to [6] where different smoothness criteria are given. The combinatorial criterion that we formulate in Theorem 2 below is a translation of [6, Theorem 1.2]. Given a standard tableau $T \in \mathbf{T a b}_{\lambda}$, let $\operatorname{col}_{1}(T)=\left\{a_{1}<\ldots<a_{n-k}\right\}$, resp. $\operatorname{col}_{2}(T)=$ $\left\{b_{1}<\ldots<b_{k}\right\}$, be the entries in its first, resp. second, column. Let $\tau^{*}(T)$ be the set of entries $i \in \operatorname{col}_{1}(T)$ such that $i+1 \in \operatorname{col}_{2}(T)$. Hereafter, by $|S|$ we denote the number of elements in a set $S$.

Theorem 2. With the above notation, the component $\mathcal{F}_{T}$ is smooth if and only if one of the following cases occurs:

(i) $\left|\tau^{*}(T)\right|=1$;

(ii) $\left|\tau^{*}(T)\right|=2$ and $\left(b_{k}=n\right.$ or there is $i \in\{1, \ldots, k\}$ such that $\left.b_{i}=2 i\right)$;

(iii) $\left|\tau^{*}(T)\right|=3$ and $\left(b_{k}=n\right.$ and there is $i \in\{1, \ldots, k-1\}$ such that $\left.b_{i}=2 i\right)$.

Example 3. If $n \leq 5$, then all the components of $\mathcal{F}_{(n-k, k) *}$ are smooth. If $n=6$, then all the components of $\mathcal{F}_{(n-k, k)^{*}}$ are smooth unless $k=2$, and the only singular component of $\mathcal{F}_{(4,2)^{*}}$ is $\mathcal{F}_{T}$ for

$$
T=\begin{array}{|l|l|}
\hline 1 & 3 \\
\hline 2 & 5 \\
\hline 4 & \multicolumn{1}{|l}{} \\
\cline { 1 - 1 } 6 &
\end{array}
$$

This first example of a singular component was already pointed out by Spaltenstein 11] and Vargas [13.

Let

$$
\operatorname{Tab}_{\lambda}^{\text {smooth }}=\left\{T \in \mathbf{T a b}_{\lambda}: \mathcal{F}_{T} \text { is smooth }\right\} .
$$

Hence, with $\lambda=(n-k, k)^{*}$ as before, $\mathbf{T a b}_{\lambda}^{\text {smooth }} \subset \mathbf{T a b}_{\lambda}$ is the subset of tableaux $T$ satisfying one of the conditions (i), (ii), (iii) in Theorem 2 In this paper, we study the structure of the components $\mathcal{F}_{T}$ for $T \in \mathbf{T a b}_{\lambda}^{\text {smooth }}$. Specifically, we are concerned with the general notions introduced in the next subsection.

2.3. Notion of iterated bundles and Poincaré polynomials. Given an algebraic projective variety $X$, let $H^{m}(X, \mathbb{Q})$ denote its rational cohomology space of degree $m$ (in the sense of sheaf cohomology). One has $H^{m}(X, \mathbb{Q})=0$ for $m \notin\{0,1, \ldots, 2 \operatorname{dim} X\}$. The numbers $h_{m}^{X}:=\operatorname{dim} H^{m}(X, \mathbb{Q})$ are called Betti numbers of $X$, the polynomial $P_{X}(t):=\sum_{m=0}^{2 \operatorname{dim} X} h_{m}^{X} t^{m}$ is called Poincaré polynomial.

These data can be computed in the case where $X$ admits a cell decomposition, that is, it can be partitioned as $X=C_{1} \sqcup \ldots \sqcup C_{k}$ with $\bigcup_{i=1}^{j} C_{i}$ closed for all $j \in\{1, \ldots, k\}$ and each $C_{i}$ isomorphic to an affine space. Then, we have $h_{m}^{X}=0$ when $m$ is odd and $h_{2 m}^{X}$ is the number of cells $C_{i}$ of dimension $m$, thus

$$
P_{X}(t)=\sum_{i=1}^{k} x^{\operatorname{dim} C_{i}} \text { where } x=t^{2} .
$$

In expressing Poincaré polynomials, the following conventions are useful. Given an integer $m \geq 0$, let $[m]_{x}=1+x+\ldots+x^{m-1}$ and $[m]_{x} !=[1]_{x} \cdots[m]_{x}$; in particular, $[0]_{x}=0$ and $[0]_{x} !=1$. Finally, for $0 \leq k \leq m$, let $\left[\begin{array}{c}m \\ k\end{array}\right]_{x}=\frac{[m]_{x} !}{[k]_{x} ![m-k]_{x} !}$ (which is a polynomial in $x$ with nonnegative integer coefficients). 
Example 4. The varieties $\mathbb{P}^{n}$ and $G r_{d}(n)$ have natural cell decompositions from which we can compute their Poincaré polynomials (cf. [3]):

$$
P_{\mathbb{P}^{n}}(t)=[n+1]_{x} \quad \text { and } \quad P_{G r_{d}(n)}(t)=\left[\begin{array}{l}
n \\
d
\end{array}\right]_{x} \quad\left(\text { where } x=t^{2}\right) .
$$

The notion of iterated bundle is defined as follows by induction. Let $B_{1}, \ldots, B_{m}$ be algebraic varieties.

- Case $m=1: X$ is an iterated bundle of base $\left(B_{1}\right)$ if there is an isomorphism $X \rightarrow B_{1}$

- Case $m \geq 2$ : $X$ is an iterated bundle of base $\left(B_{1}, \ldots, B_{m}\right)$ if there is a locally trivial fiber bundle $X \rightarrow B_{m}$ whose typical fiber is an iterated bundle of base $\left(B_{1}, \ldots, B_{m-1}\right)$.

For instance, the product $B_{1} \times \cdots \times B_{m}$ is an iterated bundle of base $\left(B_{1}, \ldots, B_{m}\right)$. In general, if $X$ is an iterated bundle of base $\left(B_{1}, \ldots, B_{m}\right)$, then its Poincaré polynomial is given by

$$
P_{X}(t)=P_{B_{1}}(t) \cdots P_{B_{m}}(t) .
$$

Example 5. It is easy to see that the flag variety $\mathcal{F}(n)$ is an iterated bundle of base $\left(\mathbb{P}^{0}, \mathbb{P}^{1}, \ldots, \mathbb{P}^{n-1}\right)$. Thus, from Example 4 and relation (2), we obtain

$$
P_{\mathcal{F}(n)}(t)=[n]_{x} ! \quad\left(\text { where } x=t^{2}\right) .
$$

We deal with a binary relation based on the notion of iterated bundles. We say that $X, Y$ have the same bundle type, and we write $X \sim_{B} Y$, if there are varieties $B_{1}, \ldots, B_{m}$ and a permutation $\sigma \in \mathfrak{S}_{m}$ such that $X$ and $Y$ are iterated bundles of bases $\left(B_{1}, \ldots, B_{m}\right)$ and $\left(B_{\sigma_{1}}, \ldots, B_{\sigma_{m}}\right)$, respectively. In particular, if $X \sim_{B} Y$, then we have $P_{X}(t)=P_{Y}(t)$.

2.4. Structure of smooth components of $\mathcal{F}_{(n-k, k)^{*}}$. As above, we fix a partition of the form $\lambda=(n-k, k)^{*}$ with $1 \leq k \leq n-k$. The next result describes the structure of any smooth component $\mathcal{F}_{T} \subset \mathcal{F}_{\lambda}$, corresponding to a standard tableau $T \in \mathbf{T a b} \mathbf{b}_{\lambda}^{\text {smooth }}$. In particular, as announced in Theorem 1 we obtain that $\mathcal{F}_{T}$ is an iterated bundle of Grassmannian varieties. Before stating the result, we introduce a piece of notation. To the tableau $T \in \mathbf{T a b}_{(n-k, k)^{*}}^{\text {smooth }}$ we associate a triple $\left(a_{T}, b_{T}, c_{T}\right)$ as follows, depending on the cardinal of $\tau^{*}(T)$ (see Theorem 2):

- If $\tau^{*}(T)=\left\{\alpha_{1}\right\}$, then set $a_{T}=\alpha_{1}-k, b_{T}=k$, and $c_{T}=n-\alpha_{1}-k$.

- If $\tau^{*}(T)=\left\{\alpha_{1}<\alpha_{2}\right\}$, then

- if $b_{k}=n$, set $a_{T}=n-k-\left(\alpha_{2}-\alpha_{1}\right), b_{T}=\alpha_{2}+k-n$, and $c_{T}=n-k-\alpha_{1}$,

- if $b_{k}<n$, set $a_{T}=n-k-\left(\alpha_{2}-\alpha_{1}\right), b_{T}=k-\alpha_{1}$, and $c_{T}=\alpha_{2}-k$, where $b_{k}$ stands for the last box in the second column of $T$.

- If $\tau^{*}(T)=\left\{\alpha_{1}<\alpha_{2}<\alpha_{3}\right\}$, then set $a_{T}=n-k-\left(\alpha_{3}-\alpha_{2}\right), b_{T}=$ $\alpha_{3}-\alpha_{1}-(n-k)$, and $c_{T}=n-k-\left(\alpha_{2}-\alpha_{1}\right)$.

As we point out in Section 3, the integers $a_{T}, b_{T}, c_{T}$ satisfy the relations

$$
a_{T} \geq 0, \quad 1 \leq b_{T} \leq k, \quad c_{T} \geq 0, \quad \text { and } \quad a_{T}+b_{T}+c_{T}=n-k .
$$

The following result is proved in Section 3 . 
Theorem 3. Let $T \in \mathbf{T a b}_{(n-k, k)^{*}}^{\text {smooth }}$ with $1 \leq k \leq n-k$. Then, the component $\mathcal{F}_{T}$ is an iterated bundle of base

$$
\begin{cases}\left(\mathcal{F}\left(a_{T}+b_{T}\right) \times \mathcal{F}\left(b_{T}+c_{T}\right), G r_{a_{T}}\left(a_{T}+c_{T}\right), \mathbb{P}^{b_{T}}, \mathbb{P}^{b_{T}+1}, \ldots, \mathbb{P}^{k-1}\right) & \text { if } b_{T}<k, \\ \left(\mathcal{F}\left(a_{T}+k\right) \times \mathcal{F}\left(k+c_{T}\right), G r_{a_{T}}\left(a_{T}+c_{T}\right)\right) & \text { if } b_{T}=k .\end{cases}
$$

In particular, we have

$$
P_{\mathcal{F}_{T}}(t)=\frac{\left[a_{T}+b_{T}\right]_{x} !\left[b_{T}+c_{T}\right]_{x} !\left[a_{T}+c_{T}\right]_{x} ![k]_{x} !}{\left[a_{T}\right]_{x} !\left[b_{T}\right]_{x} !\left[c_{T}\right]_{x} !} \text { with } x=t^{2} .
$$

Remark 1. (a) In the case where $a_{T}=0$ or $c_{T}=0$, the variety $G r_{a_{T}}\left(a_{T}+c_{T}\right)$ consists of only one point. In this case, $\mathcal{F}_{T}$ becomes an iterated bundle of base $\left(\mathcal{F}(n-k) \times \mathcal{F}\left(b_{T}\right), \mathbb{P}^{b_{T}}, \mathbb{P}^{b_{T}+1}, \ldots, \mathbb{P}^{k-1}\right)$ and its Poincaré polynomial is $P_{\mathcal{F}_{T}}(t)=$ $[n-k]_{x}$ ! [k] $]_{x}$ !. If in addition $b_{T}=k$, then we simply get $\mathcal{F}_{T} \cong \mathcal{F}(n-k) \times \mathcal{F}(k)$.

(b) As a corollary of the theorem, we obtain that every smooth component of $\mathcal{F}_{\lambda}$, for $\lambda=(n-k, k)^{*}$, admits a cell decomposition. Actually, this observation can be generalized in two ways:

- every component of $\mathcal{F}_{\lambda}$ (even singular) admits a cell decomposition, for a partition of the form $\lambda=(n-k, k)^{*}$ (see [6]);

- every smooth component of any Springer fiber $\mathcal{F}_{\lambda}$ (for any partition $\lambda \vdash$ n) admits a cell decomposition: this follows from [4, Lemma 1.3] and Bialynicki-Birula's theorem [2].

However, it is not known for $\lambda$ general if the singular components of $\mathcal{F}_{\lambda}$ can always admit a cell decomposition.

Example 6. The triples corresponding to the tableaux of Example 2 are respectively $(1,1,1),(0,1,2),(0,2,1),(1,1,1)$, and $(1,2,0)$. The corresponding components are then iterated bundles with respective bases $\left(\mathbb{P}^{1} \times \mathbb{P}^{1}, \mathbb{P}^{1}, \mathbb{P}^{1}\right),\left(\mathcal{F}(3), \mathbb{P}^{1}\right)$, $\left(\mathcal{F}(3) \times \mathbb{P}^{1}\right),\left(\mathbb{P}^{1} \times \mathbb{P}^{1}, \mathbb{P}^{1}, \mathbb{P}^{1}\right)$, and $\left(\mathcal{F}(3) \times \mathbb{P}^{1}\right)$.

2.5. Smooth components of same bundle type. In Section 4 , relying on Theorem 3, we establish the following result which classifies the components of $\mathcal{F}_{\lambda}$, for $\lambda=(n-k, k)^{*}$, in terms of their bundle type (see Subsection 2.3) and of their Poincaré polynomials.

Theorem 4. Let $T, S \in \mathbf{T a b}_{(n-k, k) *}^{\text {smooth }}$ with $1 \leq k \leq n-k$. Let $\left(a_{T}, b_{T}, c_{T}\right)$ and $\left(a_{S}, b_{S}, c_{S}\right)$ be the triples corresponding to T, $S$ in the sense of Subsection 2.4. The following conditions are equivalent:

(a) the components $\mathcal{F}_{T}$ and $\mathcal{F}_{S}$ have the same bundle type;

(b) the components $\mathcal{F}_{T}$ and $\mathcal{F}_{S}$ have the same Poincaré polynomial;

(c) either

(i) $\left(a_{T}=0\right.$ or $\left.c_{T}=0\right)$ and $\left(a_{S}=0\right.$ or $\left.c_{S}=0\right)$

or

(ii) $a_{T}, c_{T}, a_{S}, c_{S}$ are all nonzero and $\left\{a_{T}, b_{T}, c_{T}\right\}=\left\{a_{S}, b_{S}, c_{S}\right\}$ (as sets).

Example 7. In light of Example [6 we obtain that the components of $\mathcal{F}_{(3,2)^{*}}$ split into two classes: the components corresponding to the first and fourth tableaux in Example 2 have the same Poincaré polynomial $(1+x)^{4}$; the components corresponding to the second, third, and fifth tableaux in Example 2 have the Poincaré polynomial $(1+x)^{2}\left(1+x+x^{2}\right)\left(\right.$ with $\left.x=t^{2}\right)$. 


\section{Proof of Theorem 3}

Fix a partition $\lambda=(n-k, k)^{*} \vdash n$, with $1 \leq k \leq n-k$, and a smooth component $\mathcal{F}_{T} \subset \mathcal{F}_{\lambda}$. In this section, we establish the structure of iterated bundle of $\mathcal{F}_{T}$ as it is described in Theorem 3. We distinguish three cases depending on the value of $\left|\tau^{*}(T)\right|$. In each case, we first point out that the triple $\left(a_{T}, b_{T}, c_{T}\right)$ satisfies (3), as claimed in Subsection 2.4. The most elementary case is $\left|\tau^{*}(T)\right|=1$; it is treated in Subsection 3.1. For the other two cases $\left|\tau^{*}(T)\right|=2$ and $\left|\tau^{*}(T)\right|=3$, which are treated in Subsections 3.3 and 3.4, we need some preliminary facts on the relation between the structure of components and certain combinatorial algorithms, and these facts are presented in Subsection 3.2

3.1. Case $\left|\tau^{*}(T)\right|=1$. Here we assume the tableau $T \in \mathbf{T a b}_{(n-k, k)^{*}}^{\mathrm{smooth}}$ such that $\tau^{*}(T)$ consists of a single element, say $\tau^{*}(T)=\left\{\alpha_{1}\right\}$. The tableau $T$ being standard, we must have $k \leq \alpha_{1} \leq n-k$ and $T$ is actually characterized by $\alpha_{1}$ as

$$
T=\begin{array}{cc}
1 & \alpha_{1}+1 \\
\vdots & \vdots \\
\vdots & \alpha_{1}+k \\
\alpha_{1} & \\
\alpha_{1}+k+1 & \\
\vdots & \\
n &
\end{array}
$$

Note that $a_{T}=\alpha_{1}-k \geq 0, c_{T}=n-k-\alpha_{1} \geq 0$, and, evidently, $b_{T}=k \geq 1$ and $a_{T}+b_{T}+c_{T}=n-k$, hence (3) is satisfied. Let $u: V \rightarrow V$ be a nilpotent endomorphism of Jordan form $J(u)=\lambda$ so that $\mathcal{F}_{u}=\mathcal{F}_{\lambda}$; then the component $\mathcal{F}_{T} \subset \mathcal{F}_{u}$ coincides with the closure of the set $\mathcal{F}_{u}^{T}$ (see Subsection 2.1). Note that

$$
\operatorname{dim} \operatorname{Im} u=k \leq \alpha_{1} \leq n-k=\operatorname{dim} \operatorname{ker} u .
$$

The next result implies Theorem 3 in the case where $\left|\tau^{*}(T)\right|=1$.

Proposition 2. With the notation as above, we have

$$
\mathcal{F}_{T}=\left\{\left(V_{0}, \ldots, V_{n}\right) \in \mathcal{F}(V): \operatorname{Im} u \subset V_{\alpha_{1}} \subset \operatorname{ker} u\right\} .
$$

It is an iterated bundle of base $\left(\mathcal{F}\left(\alpha_{1}\right) \times \mathcal{F}\left(n-\alpha_{1}\right), G r_{\alpha_{1}-k}(n-2 k)\right)$.

Proof. Clearly, $\mathcal{X}:=\left\{\left(V_{0}, \ldots, V_{n}\right) \in \mathcal{F}(V): \operatorname{Im} u \subset V_{\alpha_{1}} \subset \operatorname{ker} u\right\}$ is a closed subvariety of $\mathcal{F}_{u}$. Let $\mathcal{Y}:=\left\{W \in G r_{\alpha_{1}}(V): \operatorname{Im} u \subset W \subset \operatorname{ker} u\right\}$. We consider the two maps

$$
\varphi: \mathcal{Y} \rightarrow G r_{\alpha_{1}-k}(\operatorname{ker} u / \operatorname{Im} u), W \mapsto W / \operatorname{Im} u
$$

and

$$
\psi: \mathcal{X} \rightarrow \mathcal{Y},\left(V_{0}, \ldots, V_{n}\right) \mapsto V_{\alpha_{1}}
$$

The map $\varphi$ is an isomorphism of varieties whereas the map $\psi$ is a locally trivial fiber bundle whose typical fiber $\psi^{-1}(W)$ is naturally isomorphic to the product of flag varieties $\mathcal{F}(W) \times \mathcal{F}(V / W) \cong \mathcal{F}\left(\alpha_{1}\right) \times \mathcal{F}\left(n-\alpha_{1}\right)$. Therefore, $\mathcal{X}$ is an iterated bundle of base $\left(\mathcal{F}\left(\alpha_{1}\right) \times \mathcal{F}\left(n-\alpha_{1}\right), G r_{\alpha_{1}-k}(n-2 k)\right)$. Then, the proof will be complete once we check that $\mathcal{X}=\mathcal{F}_{T}$.

The previous description of $\mathcal{X}$ as an iterated bundle implies in particular that $\mathcal{X}$ is irreducible. Hence, for showing the desired equality, it is sufficient to check 
the inclusion $\mathcal{F}_{T} \subset \mathcal{X}$ and, to do this, it suffices to see that we have $\mathcal{F}_{u}^{T} \subset \mathcal{X}$ (see Subsection 2.11). So, let $\left(V_{0}, \ldots, V_{n}\right) \in \mathcal{F}_{u}^{T}$ and let us check that

$$
\operatorname{Im} u \subset V_{\alpha_{1}} \subset \operatorname{ker} u \text {. }
$$

The form of the tableau $T$ and the definition of the set $\mathcal{F}_{u}^{T}$ imply that the Jordan form of the restriction $\left.u\right|_{V_{\alpha_{1}}}: V_{\alpha_{1}} \rightarrow V_{\alpha_{1}}$ is $J\left(\left.u\right|_{V_{\alpha_{1}}}\right)=\left(1^{\alpha_{1}}\right)$, which means that $\left.u\right|_{V_{\alpha_{1}}}=0$, so $V_{\alpha_{1}} \subset \operatorname{ker} u$. Similarly, the Jordan form of the restriction $\left.u\right|_{V_{\alpha_{1}+k}}$ : $V_{\alpha_{1}+k} \rightarrow V_{\alpha_{1}+k}$ is $J\left(\left.u\right|_{V_{\alpha_{1}+k}}\right)=\left(2^{k}, 1^{\alpha_{1}-k}\right)$, which implies that $\left.\operatorname{dim} \operatorname{ker} u\right|_{V_{\alpha_{1}+k}}=$ $\alpha_{1}$, so ker $\left.u\right|_{V_{\alpha_{1}+k}}=V_{\alpha_{1}}$, and $\left.\operatorname{dim} \operatorname{Im} u\right|_{V_{\alpha_{1}+k}}=k$, so $\left.\operatorname{Im} u\right|_{V_{\alpha_{1}+k}}=\operatorname{Im} u$. Using that $\left(\left.u\right|_{V_{\alpha_{1}+k}}\right)^{2}=0$, we deduce that

$$
\operatorname{Im} u=\left.\left.\operatorname{Im} u\right|_{V_{\alpha_{1}+k}} \subset \operatorname{ker} u\right|_{V_{\alpha_{1}+k}}=V_{\alpha_{1}} .
$$

The proof of the proposition is now complete.

3.2. Projection and Schützenberger involution. In this section, we consider a general partition $\lambda \vdash n$, a nilpotent endomorphism $u: V \rightarrow V$ of Jordan form $J(u)=\lambda$, and a standard tableau $T \in \mathbf{T a b}_{\lambda}$ giving rise to an irreducible component $\mathcal{F}_{T} \subset \mathcal{F}_{u}=\mathcal{F}_{\lambda}$. We consider two combinatorial procedures which transform the tableau $T$ into another tableau $T^{\prime}$ possibly of a different shape. For each procedure, we indicate a relation between the two Springer fiber components $\mathcal{F}_{T}$ and $\mathcal{F}_{T^{\prime}}$.

We call the first procedure "projection". The entries of $T$ are the numbers $1, \ldots, n$ and the entry $n$ lies in a corner of the tableau $T$. We denote by $T^{\prime}$ the subtableau of $T$ formed by the entries $1, \ldots, n-1$. Clearly, $T^{\prime}$ is a standard tableau of size $n-1$. Thus we can associate to it a Springer fiber component $\mathcal{F}_{T^{\prime}}$. We write $\lambda=\left(\lambda_{1} \geq \ldots \geq \lambda_{k}\right)$ so that $\lambda_{1}$ is the nilpotency order of $u$, i.e., $\operatorname{ker} u^{\lambda_{1}}=V \supseteq$ $\operatorname{ker} u^{\lambda_{1}-1}$. We denote by $\lambda^{*}=\left(\lambda_{1}^{*} \geq \ldots \geq \lambda_{\ell}^{*}\right)$ the lengths of the columns of $T$ (note that $\ell=\lambda_{1}$ ); in particular, $\lambda_{\ell}^{*}$ is the length of the last column, which coincides with the dimension of $V / \operatorname{ker} u^{\lambda_{1}-1}$. In the case where the entry $n$ lies in the last column of $T$, the components $\mathcal{F}_{T}$ and $\mathcal{F}_{T^{\prime}}$ are closely related. The following result is proved in [6, $\S 6.3]$.

Proposition 3. Assume that $n$ lies in the last column of $T$. Then, the image of the map

$$
\varphi: \mathcal{F}_{T} \rightarrow G r_{n-1}(V),\left(V_{0}, \ldots, V_{n}\right) \mapsto V_{n-1}
$$

is the subvariety $\mathcal{H}_{u}:=\left\{W \in G r_{n-1}(V): W \supset \operatorname{ker} u^{\lambda_{1}-1}\right\} \cong \mathbb{P}^{\lambda_{\ell}^{*}-1}$. Moreover, the map $\varphi: \mathcal{F}_{T} \rightarrow \mathcal{H}_{u}$ is a locally trivial fiber bundle with typical fiber isomorphic to $\mathcal{F}_{T^{\prime}}$. In particular, $\mathcal{F}_{T}$ is smooth if and only if $\mathcal{F}_{T^{\prime}}$ is smooth.

The second procedure is a classical algorithm due to Schützenberger, which consists of an involution $S c h: \mathbf{T a b}_{\lambda} \rightarrow \mathbf{T a b}_{\lambda}$, whose precise definition involves the procedure of "jeu de taquin". We are not going to explain the definition of the map $S c h$, one can find the details in [10, Definition 3.9.1], for example. We mention three elementary properties of the Schützenberger involution that we will need.

Lemma 1. Assume that $\lambda=(n-k, k)^{*}$ with $1 \leq k \leq n-k$ so that $T$ has two columns. Let $b_{1}<\ldots<b_{k}$ be the entries in the second column of $T$ and let $b_{1}^{\prime}<\ldots<b_{k}^{\prime}$ be the entries in the second column of $T^{\prime}:=S \operatorname{ch}(T)$.

(a) We have $\tau^{*}\left(T^{\prime}\right)=\left\{n-i: i \in \tau^{*}(T)\right\}$.

(b) There is $i \in\{1, \ldots, k\}$ such that $b_{i}=2 i$ if and only if $n$ belongs to the second column of $T^{\prime}$ (that is, $b_{k}^{\prime}=n$ ). 
(c) If $T \in \mathbf{T a b}_{\lambda}^{\text {smooth }},\left|\tau^{*}(T)\right|=2$, and $b_{k}<n$, then $T^{\prime} \in \mathbf{T a b}_{\lambda}^{\text {smooth }},\left|\tau^{*}\left(T^{\prime}\right)\right|=2$, $b_{k}^{\prime}=n$, and $\left(a_{T^{\prime}}, b_{T^{\prime}}, c_{T^{\prime}}\right)=\left(a_{T}, b_{T}, c_{T}\right)$ (with the notation of Subsection 2.4).

Proof. Part (a) is shown in [6, §4.1.3]. Part (b) is a straightforward consequence of the definition of the Schützenberger involution $S c h$ in [10, Definition 3.9.1]. As for part (c), let $T \in \mathbf{T a b}_{\lambda}^{\text {smooth }}$ such that $\tau^{*}(T)=\left\{\alpha_{1}<\alpha_{2}\right\}$ and $b_{k}<n$. Then, Theorem 2 implies that there is $i \in\{1, \ldots, k\}$ such that $b_{i}=2 i$. Invoking parts (a) and (b), we obtain that $\tau^{*}\left(T^{\prime}\right)=\left\{n-\alpha_{2}, n-\alpha_{1}\right\}, b_{k}^{\prime}=n$, and then $T^{\prime} \in \mathbf{T a b}_{\lambda}^{\text {smooth }}$ (by Theorem 21), and it is easy to see that $a_{T}=a_{T^{\prime}}, b_{T}=b_{T^{\prime}}$, and $c_{T}=c_{T^{\prime}}$.

Finally, we will use the following geometric interpretation of the Schützenberger involution in terms of Springer fiber components (see [9, §3]).

Proposition 4. Let $\lambda \vdash n, T \in \mathbf{T a b}_{\lambda}$, and $T^{\prime}=S \operatorname{ch}(T)$. Then, there is an isomorphism of varieties $\mathcal{F}_{T} \rightarrow \mathcal{F}_{T^{\prime}}$. More precisely, take $u: V \rightarrow V$ a nilpotent endomorphism of Jordan form $J(u)=\lambda$ and let $u^{*}: V^{*} \rightarrow V^{*}$ be the dual linear map, which is a nilpotent endomorphism with the same Jordan form $J\left(u^{*}\right)=\lambda$. Then, the map $\mathcal{F}_{u} \rightarrow \mathcal{F}_{u^{*}}$ given by $\left(V_{0}, \ldots, V_{n}\right) \mapsto\left(V_{n}^{\perp}, \ldots, V_{0}^{\perp}\right)$ (where $V_{i}^{\perp}:=$ $\left.\left\{v^{*} \in V^{*}: V_{i} \subset \operatorname{ker} v^{*}\right\}\right)$ is an isomorphism of varieties which restricts to an isomorphism $\mathcal{F}_{T} \stackrel{\sim}{\rightarrow} \mathcal{F}_{T^{\prime}}$.

3.3. Case $\left|\tau^{*}(T)\right|=2$. Let $T \in \mathbf{T a b}_{(n-k, k)^{*}}^{\text {smooth }}$ such that $\tau^{*}(T)=\left\{\alpha_{1}<\alpha_{2}\right\}$ and let us show that $\mathcal{F}_{T}$ is an iterated bundle with the base indicated in the statement of Theorem 3. Note that, in view of Lemma1(c) and Proposition 4, up to replacing $T$ by its Schützenberger transform $S c h(T)$, we may assume that $n$ lies in the second column of $T$. Then, applying Proposition 3, we know that $\mathcal{F}_{T}$ is an iterated bundle of base $\left(\mathcal{F}_{T^{\prime}}, \mathbb{P}^{k-1}\right)$, where we denote by $T^{\prime}$ the subtableau of $T$ formed by the entries $1, \ldots, n-1$. We distinguish two cases:

Case $1\left(\alpha_{2}=n-1\right)$. In this case, we have $\tau^{*}\left(T^{\prime}\right)=\left\{\alpha_{1}\right\}$, hence $a_{T}=a_{T^{\prime}}=$ $\alpha_{1}-(k-1), b_{T}=b_{T^{\prime}}=k-1$, and $c_{T}=c_{T^{\prime}}=n-k-\alpha_{1}$. Since $T^{\prime}$ fulfills (3) (by Subsection 3.1), it follows that (3) holds also for T. By Proposition 2, $\mathcal{F}_{T^{\prime}}$ is an iterated bundle of base $\left(\mathcal{F}\left(\alpha_{1}\right) \times \mathcal{F}\left(n-1-\alpha_{1}\right), G r_{\alpha_{1}-k+1}(n-2 k+1)\right)$. Thereby, $\mathcal{F}_{T}$ is an iterated bundle of base $\left(\mathcal{F}\left(\alpha_{1}\right) \times \mathcal{F}\left(n-1-\alpha_{1}\right), G r_{\alpha_{1}-k+1}(n-2 k+1), \mathbb{P}^{k-1}\right)$. In view of the expressions for $a_{T}, b_{T}, c_{T}$, this base conforms to the statement of Theorem 3

Case $2\left(\alpha_{2}<n-1\right)$. In this case, we have $\tau^{*}\left(T^{\prime}\right)=\left\{\alpha_{1}, \alpha_{2}\right\}$ and $n-1$ lies in the second column of $T^{\prime}$. Note that we also have $\left(a_{T^{\prime}}, b_{T^{\prime}}, c_{T^{\prime}}\right)=\left(a_{T}, b_{T}, c_{T}\right)$. It follows that $T$ fulfills (3) (because, arguing by induction, we may assume that $T^{\prime}$ fulfills (3) ). Note in particular that $b_{T}=\alpha_{2}+k-n<k-1$. Arguing by induction on $n$, we may assume that $\mathcal{F}_{T^{\prime}}$ is an iterated bundle of base

$$
\left(\mathcal{F}\left(a_{T}+b_{T}\right) \times \mathcal{F}\left(b_{T}+c_{T}\right), G r_{a_{T}}\left(a_{T}+c_{T}\right), \mathbb{P}^{b_{T}}, \mathbb{P}^{b_{T}+1}, \ldots, \mathbb{P}^{k-2}\right) .
$$

This implies that $\mathcal{F}_{T}$ is an iterated bundle with the desired base

$$
\left(\mathcal{F}\left(a_{T}+b_{T}\right) \times \mathcal{F}\left(b_{T}+c_{T}\right), G r_{a_{T}}\left(a_{T}+c_{T}\right), \mathbb{P}^{b_{T}}, \mathbb{P}^{b_{T}+1}, \ldots, \mathbb{P}^{k-1}\right),
$$

which completes the proof of the theorem in the case where $\left|\tau^{*}(T)\right|=2$. 
3.4. Case $\left|\tau^{*}(T)\right|=3$. Let $T \in \operatorname{Tab}_{(n-k, k)^{*}}^{\text {smooth }}$ such that $\tau^{*}(T)=\left\{\alpha_{1}<\alpha_{2}<\alpha_{3}\right\}$. By Theorem 2, $n$ lies in the second column of $T$. Hence, applying Proposition 3, we obtain that $\mathcal{F}_{T}$ is an iterated bundle of base $\left(\mathcal{F}_{T^{\prime}}, \mathbb{P}^{k-1}\right)$, where $T^{\prime}$ is the subtableau of $T$ formed by the entries $1, \ldots, n-1$. As in Subsection 3.3 , we distinguish two cases:

Case $1\left(\alpha_{3}=n-1\right)$. In this case, we have $\tau^{*}\left(T^{\prime}\right)=\left\{\alpha_{1}, \alpha_{2}\right\}$ and $n-1$ lies in the first column of $T^{\prime}$. Hence $a_{T^{\prime}}=n-k-\left(\alpha_{2}-\alpha_{1}\right)=c_{T}, b_{T^{\prime}}=k-1-\alpha_{1}=b_{T}$ (which is $<k-1$ ), and $c_{T^{\prime}}=\alpha_{2}-k+1=a_{T}$. From these equalities, we deduce that $T$ fulfills (3) (because we know from Subsection 3.3 that (3) holds for $T^{\prime}$ ). By Subsection 3.3 , we know that $\mathcal{F}_{T^{\prime}}$ is an iterated bundle of base

$$
\left(\mathcal{F}\left(c_{T}+b_{T}\right) \times \mathcal{F}\left(b_{T}+a_{T}\right), G r_{c_{T}}\left(a_{T}+c_{T}\right), \mathbb{P}^{b_{T}}, \mathbb{P}^{b_{T}+1}, \ldots, \mathbb{P}^{k-2}\right) .
$$

Note that $G r_{c_{T}}\left(a_{T}+c_{T}\right) \cong G r_{a_{T}}\left(a_{T}+c_{T}\right)$. We conclude that $\mathcal{F}_{T}$ is an iterated bundle of base

$$
\left(\mathcal{F}\left(a_{T}+b_{T}\right) \times \mathcal{F}\left(b_{T}+c_{T}\right), G r_{a_{T}}\left(a_{T}+c_{T}\right), \mathbb{P}^{b_{T}}, \mathbb{P}^{b_{T}+1}, \ldots, \mathbb{P}^{k-1}\right),
$$

as stated in Theorem 3

Case $2\left(\alpha_{3}<n-1\right)$. In this case, we have $\tau^{*}\left(T^{\prime}\right)=\left\{\alpha_{1}, \alpha_{2}, \alpha_{3}\right\}$ and $n-1$ lies in the second column of $T^{\prime}$. Clearly, $\left(a_{T^{\prime}}, b_{T^{\prime}}, c_{T^{\prime}}\right)=\left(a_{T}, b_{T}, c_{T}\right)$ and $b_{T^{\prime}}=b_{T}<k-2$. Thereby (3) holds for $T$ (since we can assume (arguing by induction) that it holds for $T^{\prime}$ ). Arguing by induction on $n$, we may also assume that $\mathcal{F}_{T^{\prime}}$ fulfills Theorem 3. that is, $\mathcal{F}_{T^{\prime}}$ is an iterated bundle of base

$$
\left(\mathcal{F}\left(a_{T}+b_{T}\right) \times \mathcal{F}\left(b_{T}+c_{T}\right), G r_{a_{T}}\left(a_{T}+c_{T}\right), \mathbb{P}^{b_{T}}, \mathbb{P}^{b_{T}+1}, \ldots, \mathbb{P}^{k-2}\right) .
$$

Therefore, $\mathcal{F}_{T}$ is an iterated bundle of base

$$
\left(\mathcal{F}\left(a_{T}+b_{T}\right) \times \mathcal{F}\left(b_{T}+c_{T}\right), G r_{a_{T}}\left(a_{T}+c_{T}\right), \mathbb{P}^{b_{T}}, \mathbb{P}^{b_{T}+1}, \ldots, \mathbb{P}^{k-1}\right),
$$

as desired, so that the proof of Theorem 3 is now complete.

\section{Proof of Theorem 4}

In this last section, we establish the equivalence between the assertions (a), (b), and $(c)$ of Theorem 4 Note that the implication $(a) \Rightarrow(b)$ is immediate. In the following subsections, we prove the implications $(\mathrm{c}) \Rightarrow(\mathrm{a})$ and $(\mathrm{b}) \Rightarrow(\mathrm{c})$.

4.1. Proof of the implication $(\mathrm{c}) \Rightarrow(\mathrm{a})$. We rely on the following lemma:

Lemma 2. For integers $a, b \geq 0$, the flag variety $\mathcal{F}(a+b)$ is an iterated bundle of base $\left(\mathcal{F}(a) \times \mathcal{F}(b), G r_{a}(a+b)\right)$.

Proof. The map $\varphi: \mathcal{F}(a+b) \rightarrow G r_{a}(a+b),\left(V_{0}, \ldots, V_{a+b}\right) \mapsto V_{a}$ is a locally trivial fiber bundle and, given $W \in G r_{a}(a+b)$, the fiber $\varphi^{-1}(W)$ is isomorphic to $\mathcal{F}(W) \times \mathcal{F}\left(\mathbb{C}^{a+b} / W\right)$, hence, to $\mathcal{F}(a) \times \mathcal{F}(b)$.

Let $T \in \mathbf{T a b}_{(n-k, k)^{*}}^{\text {smooth }}$. Combining Theorem 3, Lemma 2, Example 5] and using also the fact that $G r_{a}(a+b) \cong G r_{b}(a+b)$ whenever $a, b \geq 0$, we obtain that $\mathcal{F}_{T}$ is an iterated bundle whose base is a permutation of the sequence

$$
\left(\mathcal{F}\left(a_{T}\right), \mathcal{F}\left(b_{T}\right), \mathcal{F}\left(c_{T}\right), G r_{\tilde{c}_{T}}\left(a_{T}+b_{T}\right), G r_{\tilde{b}_{T}}\left(a_{T}+c_{T}\right), G r_{\tilde{a}_{T}}\left(b_{T}+c_{T}\right), \mathbb{P}^{0}, \mathbb{P}^{1}, \ldots, \mathbb{P}^{k-1}\right)
$$

where we write $\tilde{c}_{T}=\min \left\{a_{T}, b_{T}\right\}, \tilde{b}_{T}=\min \left\{a_{T}, c_{T}\right\}$, and $\tilde{a}_{T}=\min \left\{b_{T}, c_{T}\right\}$. In this way, it appears that, up to permutation, this base only depends on the set 
$\left\{a_{T}, b_{T}, c_{T}\right\}$. This guarantees that $\mathcal{F}_{T}$ and $\mathcal{F}_{S}$ have the same bundle type whenever $\left\{a_{T}, b_{T}, c_{T}\right\}=\left\{a_{S}, b_{S}, c_{S}\right\}$.

Now, assume that we have $a_{T}=0$ or $c_{T}=0$. Then, Remark 1.(a) and Example [5] imply that $\mathcal{F}_{T}$ is an iterated bundle of base

$$
\left(\mathcal{F}(n-k), \mathbb{P}^{0}, \mathbb{P}^{1}, \ldots, \mathbb{P}^{k-1}\right)
$$

and this sequence is independent of the triple $\left(a_{T}, b_{T}, c_{T}\right)$. Whence, we obtain that $\mathcal{F}_{T}$ and $\mathcal{F}_{S}$ have the same bundle type whenever $\left(a_{T}, b_{T}, c_{T}\right)$ and $\left(a_{S}, b_{S}, c_{S}\right)$ are triples such that $\left(a_{T}=0\right.$ or $\left.c_{T}=0\right)$ and $\left(a_{S}=0\right.$ or $\left.c_{S}=0\right)$.

We have shown the implication $(\mathrm{c}) \Rightarrow(\mathrm{a})$.

4.2. Proof of the implication $(b) \Rightarrow(c)$. In view of Theorem 3 , we only need to show the following proposition.

Proposition 5. Let $a_{1}, a_{2}, a_{3}, b_{1}, b_{2}, b_{3}$ be nonnegative integers such that $a_{1}+a_{2}+$ $a_{3}=b_{1}+b_{2}+b_{3}$ and let

$$
P(x)=\frac{\left[a_{1}+a_{2}\right]_{x} !\left[a_{1}+a_{3}\right]_{x} !\left[a_{2}+a_{3}\right]_{x} !}{\left[a_{1}\right]_{x} !\left[a_{2}\right]_{x} !\left[a_{3}\right]_{x} !} \text { and } Q(x)=\frac{\left[b_{1}+b_{2}\right]_{x} !\left[b_{1}+b_{3}\right]_{x} !\left[b_{2}+b_{3}\right]_{x} !}{\left[b_{1}\right]_{x} !\left[b_{2}\right]_{x} !\left[b_{3}\right]_{x} !} \text {. }
$$

(a) Assume that $\min \left\{a_{1}, a_{2}, a_{3}\right\}=0$ and $\min \left\{b_{1}, b_{2}, b_{3}\right\}>0$. Then, $P(x) \neq Q(x)$.

(b) Assume that $a_{1}, a_{2}, a_{3}, b_{1}, b_{2}, b_{3}$ are all positive, but $\left\{a_{1}, a_{2}, a_{3}\right\} \neq\left\{b_{1}, b_{2}, b_{3}\right\}$. Then, $P(x) \neq Q(x)$.

The remainder of the section is devoted to the proof of Proposition 5 . We rely on a partial order on polynomials called domination: given $f(x), g(x) \in \mathbb{R}[x]$, we write

$$
f(x) \preceq g(x) \text { if } f^{(\ell)}(0) \leq g^{(\ell)}(0) \text { for all } \ell \geq 0,
$$

where $f^{(\ell)}, g^{(\ell)}$ stand for the $\ell$-th derivatives. Equivalently, letting $f(x)=\sum_{\ell \geq 0} a_{\ell} x^{\ell}$ and $g(x)=\sum_{\ell \geq 0} b_{\ell} x^{\ell}$, we have $f(x) \preceq g(x)$ if and only if $a_{\ell} \leq b_{\ell}$ for all $\ell \geq 0$. In particular, we have $f(x) \succeq 0$ if and only if all the coefficients of $f(x)$ are nonnegative; for such polynomials, we note in the following lemma that the domination is preserved by multiplication. For products of polynomials of the type $[a]_{x}$, we can deduce a stronger property, also explained in the next lemma.

Lemma 3. (a) Let $f_{1}(x), f_{2}(x), g_{1}(x), g_{2}(x) \in \mathbb{R}[x]$. If $0 \preceq f_{1}(x) \preceq g_{1}(x)$ and $0 \preceq f_{2}(x) \preceq g_{2}(x)$, then $f_{1}(x) f_{2}(x) \preceq g_{1}(x) g_{2}(x)$, with equality if and only if $g_{1}(x)=0$ or $g_{2}(x)=0$ or $\left(f_{1}(x)=g_{1}(x)\right.$ and $\left.f_{2}(x)=g_{2}(x)\right)$.

(b) Let $a, b, c, d$ be integers such that $1 \leq a \leq b \leq c \leq d$ and $a+d \leq b+c$. Then, $[a]_{x}[d]_{x} \preceq[b]_{x}[c]_{x}$, with equality if and only if $a=b$.

Proof. (a) For integers $0 \leq h \leq \ell$, we have $f_{1}^{(h)}(0) f_{2}^{(\ell-h)}(0) \leq g_{1}^{(h)}(0) g_{2}^{(\ell-h)}(0)$, hence, by Leibniz's formula, we obtain

$$
\left(f_{1} f_{2}\right)^{(\ell)}(0)=\sum_{h=0}^{\ell}\left(\begin{array}{l}
\ell \\
h
\end{array}\right) f_{1}^{(h)}(0) f_{2}^{(\ell-h)}(0) \leq \sum_{h=0}^{\ell}\left(\begin{array}{l}
\ell \\
h
\end{array}\right) g_{1}^{(h)}(0) g_{2}^{(\ell-h)}(0)=\left(g_{1} g_{2}\right)^{(\ell)}(0),
$$

whence $f_{1}(x) f_{2}(x) \preceq g_{1}(x) g_{2}(x)$. For the equality case, it is sufficient to note that, if $g_{1}(x), g_{2}(x) \neq 0$ and, say, $f_{1}(x) \prec g_{1}(x)$, then we can find $\ell, h$ with $0 \leq h \leq \ell$ such that $f_{1}^{(h)}(0)<g_{1}^{(h)}(0)$ and $g_{2}^{(\ell-h)}(0)>0$, thus $f_{1}^{(h)}(0) f_{2}^{(\ell-h)}(0)<g_{1}^{(h)}(0) g_{2}^{(\bar{\ell}-h)}(0)$.

(b) First, for $\ell \geq 0$, we note that

$$
[a]_{x}^{(\ell)}(0)= \begin{cases}\ell ! & \text { if } \ell<a \\ 0 & \text { if } \ell \geq a .\end{cases}
$$


Let $f(x)=[a]_{x}[d]_{x}$. By Leibniz's formula, we have

$$
f^{(\ell)}(0)=\sum_{h=0}^{\ell}\left(\begin{array}{l}
\ell \\
h
\end{array}\right)[a]_{x}^{(h)}(0)[d]_{x}^{(\ell-h)}(0),
$$

which yields

$$
f^{(\ell)}(0)= \begin{cases}(\ell+1) ! & \text { if } \ell<a \\ a \ell ! & \text { if } a \leq \ell<d \\ (a+d-\ell-1) \ell ! & \text { if } d \leq \ell<a+d \\ 0 & \text { if } \ell \geq a+d .\end{cases}
$$

Exactly in the same way, for $g(x):=[b]_{x}[c]_{x}$, we have

$$
g^{(\ell)}(0)= \begin{cases}(\ell+1) ! & \text { if } \ell<b \\ b \ell ! & \text { if } b \leq \ell<c \\ (b+c-\ell-1) \ell ! & \text { if } c \leq \ell<b+c \\ 0 & \text { if } \ell \geq b+c .\end{cases}
$$

The conclusion follows by comparing both formulas.

We are now in position to prove Proposition 5 .

Proof. Let $\left(a_{1}, a_{2}, a_{3}\right)$ and $\left(b_{1}, b_{2}, b_{3}\right)$ be triples of positive integers satisfying the conditions of Proposition 5. Set $m=a_{1}+a_{2}+a_{3}=b_{1}+b_{2}+b_{3}$. Note that the polynomials involved in the statement of Proposition 5 can be rewritten under the form

$$
P(x)=\prod_{i=1}^{a_{1}}\left[a_{2}+i\right]_{x} \prod_{i=1}^{a_{2}}\left[a_{3}+i\right]_{x} \prod_{i=1}^{a_{3}}\left[a_{1}+i\right]_{x}
$$

and

$$
Q(x)=\prod_{i=1}^{b_{1}}\left[b_{2}+i\right]_{x} \prod_{i=1}^{b_{2}}\left[b_{3}+i\right]_{x} \prod_{i=1}^{b_{3}}\left[b_{1}+i\right]_{x} .
$$

(a) We assume that $\min \left\{a_{1}, a_{2}, a_{3}\right\}=0<\min \left\{b_{1}, b_{2}, b_{3}\right\}$. In this situation, we will show that $P(x) \prec Q(x)$, which yields in particular $P(x) \neq Q(x)$. Up to re-indexing the triples, we may assume without loss of generality that

$$
a_{1}=0 \leq a_{2} \leq a_{3} \text { and } 0<b_{1} \leq b_{2} \leq b_{3} .
$$

Then, we can rewrite

$$
P(x)=[m]_{x} !=R(x) \prod_{i=1}^{b_{1}}[i]_{x}[m-i+1]_{x}
$$

and

$$
Q(x)=R(x) \prod_{i=1}^{b_{1}}\left[b_{2}+i\right]_{x}\left[b_{1}+b_{3}-i+1\right]_{x},
$$

where $R(x)=\left[b_{1}+1\right]_{x} \cdots\left[m-b_{1}\right]_{x}$. For all $i \in\left\{1, \ldots, b_{1}\right\}$, we have

$$
i<\min \left\{b_{2}+i, b_{1}+b_{3}-i+1\right\} \leq \max \left\{b_{2}+i, b_{1}+b_{3}-i+1\right\}<m-i+1
$$

and

$$
i+(m-i+1)=\left(b_{2}+i\right)+\left(b_{1}+b_{3}-i+1\right) .
$$


Thus, by Lemma 3(b) and (a), we get

$$
\prod_{i=1}^{b_{2}}[i]_{x}[m-i+1]_{x} \prec \prod_{i=1}^{b_{1}}\left[b_{2}+i\right]_{x}\left[b_{1}+b_{3}-i+1\right]_{x} .
$$

Combining (6), (7), (8) with Lemma 3(a), we conclude that $P(x) \prec Q(x)$.

(b) Up to re-indexing the triples, it suffices to consider the two situations corresponding to Cases 1 and 2 below.

Case $1\left(a_{1}=b_{1}\right.$ and $\left.a_{2}>b_{2} \geq b_{3}>a_{3}\right)$.

Case $2\left(a_{1} \geq a_{2} \geq a_{3}, b_{1} \geq b_{2} \geq b_{3}>a_{3}\right.$, and $\left.\left\{a_{1}, a_{2}, a_{3}\right\} \cap\left\{b_{1}, b_{2}, b_{3}\right\}=\emptyset\right)$.

In Case 1 , we can show that $P(x) \prec Q(x)$. We distinguish two subcases.

Subcase $1.1\left(a_{1}=b_{1} \leq b_{2}\right.$ and $\left.a_{2}>b_{2} \geq b_{3}>a_{3}\right)$. In this subcase, we can write

$P(x)=\prod_{i=1}^{p}\left[a_{1}+i\right]_{x} \prod_{i=1}^{a_{1}}\left[a_{2}+i\right]_{x}\left[a_{3}+i\right]_{x}$ and $Q(x)=\prod_{i=1}^{p}\left[a_{1}+i\right]_{x} \prod_{i=1}^{a_{1}}\left[b_{2}+i\right]_{x}\left[b_{3}+i\right]_{x}$,

where $p:=a_{2}+a_{3}-a_{1}=b_{2}+b_{3}-b_{1} \geq 1$. Moreover, since $a_{2}+a_{3}=b_{2}+b_{3}$ and $a_{2}>b_{2} \geq b_{3}>a_{3}$, invoking Lemma 3(b), we have

$$
\left[a_{2}+i\right]_{x}\left[a_{3}+i\right]_{x} \prec\left[b_{2}+i\right]_{x}\left[b_{3}+i\right]_{x} \text { for all integer } i \geq 1,
$$

hence, by Lemma 3(a) (and since $a_{1} \geq 1$ ), we obtain

$$
\prod_{i=1}^{a_{1}}\left[a_{2}+i\right]_{x}\left[a_{3}+i\right]_{x} \prec \prod_{i=1}^{a_{1}}\left[b_{2}+i\right]_{x}\left[b_{3}+i\right]_{x} .
$$

Combining (9), (10), and Lemma 3(a), we conclude that $P(x) \prec Q(x)$.

Subcase $1.2\left(a_{1}=b_{1}>b_{2}\right.$ and $\left.a_{2}>b_{2} \geq b_{3}>a_{3}\right)$. Combining these assumptions with the relation $a_{1}+a_{2}+a_{3}=b_{1}+b_{2}+b_{3}$, we obtain

$$
0<a_{2}-b_{2}=b_{3}-a_{3} \leq b_{3}<b_{1}=a_{1} \text { and } 0<b_{3}-a_{3}=a_{2}-b_{2}<a_{2} .
$$

Using this fact, from (4) and (5), we can rewrite $P(x)$ and $Q(x)$ as

$$
\begin{aligned}
P(x) & =\prod_{i=1}^{a_{1}+b_{2}-a_{2}}\left[a_{2}+i\right]_{x} \prod_{i=a_{1}+b_{2}-a_{2}+1}^{a_{1}}\left[a_{2}+i\right]_{x} \prod_{i=1}^{b_{3}-a_{3}}\left[a_{3}+i\right]_{x} \prod_{i=b_{3}-a_{3}+1}^{a_{2}}\left[a_{3}+i\right]_{x} \prod_{i=1}^{a_{3}}\left[a_{1}+i\right]_{x} \\
& =S(x) \prod_{i=1}^{a_{2}-b_{2}}\left[a_{1}+b_{2}+i\right]_{x}\left[a_{3}+i\right]_{x}
\end{aligned}
$$

and

$$
\begin{aligned}
Q(x) & =\prod_{i=1}^{a_{2}-b_{2}}\left[b_{2}+i\right]_{x} \prod_{i=a_{2}-b_{2}+1}^{b_{1}}\left[b_{2}+i\right]_{x} \prod_{i=1}^{b_{2}}\left[b_{3}+i\right]_{x} \prod_{i=1}^{a_{3}}\left[b_{1}+i\right]_{x} \prod_{i=a_{3}+1}^{b_{3}}\left[b_{1}+i\right]_{x} \\
& =S(x) \prod_{i=1}^{a_{2}-b_{2}}\left[b_{2}+i\right]_{x}\left[a_{1}+a_{3}+i\right]_{x}
\end{aligned}
$$

where

$$
S(x)=\prod_{i=1}^{a_{1}+b_{2}-a_{2}}\left[a_{2}+i\right]_{x} \prod_{i=1}^{b_{2}}\left[b_{3}+i\right]_{x} \prod_{i=1}^{a_{3}}\left[a_{1}+i\right]_{x}
$$


By Lemma 3(a), for proving that $P(x) \prec Q(x)$, it now suffices to check the relation

$$
\left[a_{1}+b_{2}+i\right]_{x}\left[a_{3}+i\right]_{x} \prec\left[b_{2}+i\right]_{x}\left[a_{1}+a_{3}+i\right]_{x} \text { for all } i \in\left\{1, \ldots, a_{2}-b_{2}\right\} .
$$

Since our assumptions imply that we have $a_{3}<b_{2}<a_{1}+a_{3}<a_{1}+b_{2}$, relation (11) is obtained by applying Lemma 3(b). The proof is complete in Case 1 .

Now, we deal with Case 2. In this case, we use comparison modulo. Given integers $i, j \geq 0$, we note that

$$
[i]_{x}= \begin{cases}{[i]_{x}\left(\bmod x^{j}\right)} & \text { if } i<j, \\ {[j]_{x}\left(\bmod x^{j}\right)} & \text { if } i \geq j .\end{cases}
$$

Applying this rule, from (44) and (5), we obtain that

$$
P(x)= \begin{cases}{\left[a_{3}+1\right]_{x}\left[a_{3}+2\right]_{x}^{m-1}\left(\bmod x^{a_{3}+2}\right)} & \text { if } a_{3}<a_{2}, \\ {\left[a_{3}+1\right]_{x}^{2}\left[a_{3}+2\right]_{x}^{m-2}\left(\bmod x^{a_{3}+2}\right)} & \text { if } a_{3}=a_{2},\end{cases}
$$

whereas

$$
Q(x)=\left[a_{3}+2\right]_{x}^{m}\left(\bmod x^{a_{3}+2}\right),
$$

where $m=a_{1}+a_{2}+a_{3}=b_{1}+b_{2}+b_{3}$ as before. It follows that $P(x) \neq Q(x)$ $\left(\bmod x^{a_{3}+2}\right)$, so that $P(x) \neq Q(x)$. The proof is complete in Case 2, too.

Remark 2. Given a triple of nonnegative integers $\left(a_{1}, a_{2}, a_{3}\right)$, let $P_{\left(a_{1}, a_{2}, a_{3}\right)}(x)=$ $P(x)$ be the corresponding polynomial in the sense of Proposition [5. Given $m \geq 0$, let $\mathcal{P}_{m}$ be the set of polynomials of the form $P_{\left(a_{1}, a_{2}, a_{3}\right)}(x)$ for a triple $\left(a_{1}, a_{2}, a_{3}\right)$ such that $m=a_{1}+a_{2}+a_{3}$. In general, the order $\preceq$ is not a total order on the set $\mathcal{P}_{m}$. For example, for $m=16$, one can see that $P_{(2,6,8)}^{(3)}(0)<P_{(3,3,10)}^{(3)}(0)$ but $P_{(2,6,8)}(1)>P_{(3,3,10)}(1)$ so that it is impossible to have either $P_{(2,6,8)} \prec P_{(3,3,10)}$ or $P_{(3,3,10)} \prec P_{(2,6,8)}$. Nevertheless, in view of the proof of Proposition 5 (specifically, the proof of part (a), and Case 1 in the proof of part (b)), we see that $\preceq$ becomes a total order when restricted to the subset of polynomials with one fixed entry, namely $\mathcal{P}_{m, c}:=\left\{P_{\left(a_{1}, a_{2}, a_{3}\right)}(x) \in \mathcal{P}_{m}: c \in\left\{a_{1}, a_{2}, a_{3}\right\}\right\}$, for $0 \leq c \leq m$.

\section{REFERENCES}

[1] L. Barchini and R. Zierau, Certain components of Springer fibers and associated cycles for discrete series representations of $\mathrm{SU}(p, q)$, Represent. Theory 12 (2008), 403-434, DOI 10.1090/S1088-4165-08-00311-7. With an appendix by Peter E. Trapa. MR2461236 (2009h:22009)

[2] A. Białynicki-Birula, Some theorems on actions of algebraic groups, Ann. of Math. (2) 98 (1973), 480-497. MR0366940 (51 \#3186)

[3] Michel Brion, Lectures on the geometry of flag varieties, Topics in cohomological studies of algebraic varieties, Trends Math., Birkhäuser, Basel, 2005, pp. 33-85, DOI 10.1007/3-76437342-3_2. MR2143072 (2006f:14058)

[4] Lucas Fresse, Singular components of Springer fibers in the two-column case (English, with English and French summaries), Ann. Inst. Fourier (Grenoble) 59 (2009), no. 6, 2429-2444. MR2640925 (2011f:14081)

[5] Lucas Fresse, On the singularity of some special components of Springer fibers, J. Lie Theory 21 (2011), no. 1, 205-242. MR2797827 (2012c:14099)

[6] Lucas Fresse and Anna Melnikov, Some characterizations of singular components of Springer fibers in the two-column case, Algebr. Represent. Theory 14 (2011), no. 6, 1063-1086, DOI 10.1007/s10468-010-9227-5. MR2844756 (2012m:14087)

[7] Francis Y. C. Fung, On the topology of components of some Springer fibers and their relation to Kazhdan-Lusztig theory, Adv. Math. 178 (2003), no. 2, 244-276, DOI 10.1016/S00018708(02)00072-5. MR1994220 (2004m:20087) 
[8] William Graham and R. Zierau, Smooth components of Springer fibers (English, with English and French summaries), Ann. Inst. Fourier (Grenoble) 61 (2011), no. 5, 2139-2182 (2012), DOI 10.5802/aif.2669. MR2961851

[9] Marc A. A. van Leeuwen, Flag varieties and interpretations of Young tableau algorithms, J. Algebra 224 (2000), no. 2, 397-426, DOI 10.1006/jabr.1999.8070. MR1739585(2001h:14063)

[10] Bruce E. Sagan, The symmetric group, 2nd ed., Graduate Texts in Mathematics, vol. 203, Springer-Verlag, New York, 2001. Representations, combinatorial algorithms, and symmetric functions. MR 1824028(2001m:05261)

[11] Nicolas Spaltenstein, Classes unipotentes et sous-groupes de Borel (French), Lecture Notes in Mathematics, vol. 946, Springer-Verlag, Berlin, 1982. MR672610 (84a:14024)

[12] T. A. Springer, The unipotent variety of a semi-simple group, Algebraic Geometry (Internat. Colloq., Tata Inst. Fund. Res., Bombay, 1968), Oxford Univ. Press, London, 1969, pp. 373391. MR0263830(41 \#8429)

[13] J. A. Vargas, Fixed points under the action of unipotent elements of $\mathrm{SL}_{n}$ in the flag variety, Bol. Soc. Mat. Mexicana (2) 24 (1979), no. 1, 1-14. MR579665 (81h:14027)

Université de Lorraine, CNRS, institut Élie Cartan de Lorraine, UMR 7502, VanDOEUVRE-LÈs-NANCY, F-54506, FranCE

E-mail address: lucas.fresse@univ-lorraine.fr

Department of Mathematics, University of Haifa, Haifa 31905, Israel

E-mail address: melnikov@math.haifa.ac.il

Department of Mathematics, University of Haifa, Haifa 31905, Israel

E-mail address: obsamar@gmail.com 